\title{
CLASSIFICATION OF HOMOTOPY TORUS KNOT SPACES
}

\author{
RICHARD S. STEVENS
}

ABSTRACT. The existence of nontrivial homotopy torus knot spaces is established as a corollary to the

Theorem. Let $p$ and $q$ be two integers with $p>1, q>1$, and $(p, q)$ $=1$. Let $\pi$ be a maximal set of topologically distinct compact orientable irreducible 3-mainfolds with fundamental group presented by $\left\langle a, b \mid a^{p} b^{q}\right\rangle$. Then $\operatorname{card}(\pi)=1 / 2 \Phi(p q)$, where $\Phi$ denotes Euler's function.

All spaces are piecewise linear. The symbols $I, D$, and $B$ denote the closed unit interval, disc, and ball, respectively. $S^{i}$ denotes the $i$-dimensional sphere; $i=1,2,3$. The closure and boundary of a space $X$ are denoted respectively by $\mathrm{cl}(X)$ and $\partial X$. The term knot space refers to the closure of the complement in $S^{3}$ of a regular neighborhood of a knot. If $m$ and $n$ are positive integers and $(m, n)=1$, then the torus knot space corresponding to the pair $(m, n)$ is denoted by $K t(m, n)$.

A 3-mainfold is irreducible if every embedded 2-sphere bounds a 3-cell. Let $M$ be a compact manifold with boundary and $K$ be a 2 -complex in $M$. If $M-K$ is homeomorphic to $\partial M \times(0,1]$, then $K$ is called a spine of $M$. If $M$ has empty boundary and $K$ is a spine of $\operatorname{cl}(M-B)$, then we will say that $K$ is a spine of $M$.

If $M_{1}$ and $M_{2}$ are compact 3-manifolds with boundary, $M_{1} \subset M_{2}$, $\operatorname{cl}\left(M_{2}-M_{1}\right)=U=D \times I$, and $M_{1} \cap U=\partial D \times I$, then we will say that $M_{2}$ is obtained from $M_{1}$ by attaching the 2-handle $U$ to $M_{1}$. If $\gamma$ is a simple closed curve in $\partial M_{1}$ having $\partial D \times I$ as a regular neighborhood in $\partial M_{1}$, then we will say that $U$ was attached to $M_{1}$ along $\gamma$.

We distinguish between the terms group and group presentation. If $\psi=$ $\left\langle x_{1}, \ldots, x_{m} \mid R_{1}, \ldots, R_{n}\right\rangle$ is a group presentation, then $G_{\psi}$ denotes the group presented by $\psi, K_{\psi}$ the 2-complex corresponding to $\psi$, and $P_{\psi}$ the corresponding $P$-graph (see [4]). Note that if $K_{\psi}$ is a spine of the compact 3manifold $M$, then $\pi_{1}(M)=G_{\psi}$.

Henceforth $\psi$ denotes the presentation $\left\langle a, b \mid a^{p} b^{q}\right\rangle$.

Lemma. Under the conditions of the Theorem, if $M \in \mathbb{M}$ then $K_{\psi}$ is a spine of $M$.

Proof. By results of Waldhausen [5], $M$ is a Seifert fiber space with

Received by the editors June 24, 1974.

AMS (MOS) subject classifications (1970). Primary 57A10; Secondary 55A25.

Key words and phrases. Compact orientable 3-mainfold, lens space, Seifert fiber space, spine, 2-complex corresponding to group presentation. 
orientable quotient surface $E$, and $\pi_{1}(M)$ is presented by

$$
\psi^{*}=\left\langle x_{1}, \ldots, x_{?_{g}{ }_{r}}, y_{1}, \ldots, y_{s}, z \mid\left[x_{i}, z\right]=1, y_{i}^{\mu_{i}}=z\right\rangle,
$$

where the genus of $E$ is $g \geqq 0$, where $\partial M$ has $r+1>0$ components, each component a torus $S^{1} \times S^{1}$, and where $M$ has $s \geqq 0$ exceptional fibers with respective orders $\mu_{1}, \ldots, \mu_{s}$. Since $G_{\psi}$ is isomorphic to $G_{\psi *}$, we infer that $r=g=0, s=2$, and that $\mu_{1}$ and $\mu_{2}$ are $p$ and $q$ in some order. This is argued by considering $\pi_{1}(M)$ modulo its center and applying results of [2, \$4.1].

It now follows that $E$ is a disc with two exceptional points $x_{1}$ and $x_{2}$ corresponding to the two exceptional fibers. Let $\gamma$ be an arc in $E$ with $\partial y$ $=\gamma \cap \partial E$ and $\gamma$ separating $x_{1}$ from $x_{2}$. Let $F$ be the union of all fibers projecting onto $\gamma$. Then $F$ is an annulus that separates $M$ into two solid tori $T_{1}$ and $T_{2}$. Write $F=S^{1} \times I$ and consider the arc $\delta=\{t\} \times I$ in $M$, where $t \in S^{1}$. Note that $\partial \delta=\delta \cap \partial M$. Let $U$ be a regular neighborhood of $\delta$. Then $\mathrm{cl}(M-U)$ is a genus 2 handlebody to which the 2-handle $U$ is attached. Clearly $U \cap T_{1}$ and $U \cap T_{2}$ are each connected. Thus $U$ is attached to $T$ according to the word $a^{p} b^{q}$, and the Lemma follows

Proof of the Theorem. Choose $r$ and $s$ so that $(r, p)=1 \leqq r<p$ and $(s, q)=1 \leqq s<q$. By the results of [3], we know that a compact orientable 3-manifold $M_{p, r, q, s}$ with boundary and with spine $K_{\psi}$ is uniquely determined by the faithful embedding of $P_{\psi}$ in $S^{2}$ with gap $r$ on the a-syllable graph and gap $s$ on the $b$-syllable graph. Any such manifold, having $K_{\psi}$ as a spine, is thus of the same homotopy type as $K t(p, q)$. It will suffice to show that $M_{p, r, q, s}$ and $M_{p, r^{\prime}, q, s^{\prime}}$ are homeomorphic if and only if $r \equiv \epsilon r^{\prime}(\bmod p)$ and $s \equiv \epsilon S^{\prime}(\bmod q)$ where $\epsilon= \pm 1$.

The if part is clear; if $\epsilon=-1$, then we merely reverse the orientation.

Let $T$ denote a genus 2 handlebody with inner meridian discs corresponding to the generators $a$ and $b$. Following the construction of $M=M_{p, r, q, s}$, the above-mentioned faithful embedding of $P_{\psi}$ determines a simple closed curve $\gamma_{0}$ (corresponding to the word $a^{p} b^{q}$ ) in $\partial T$. Then $M$ is obtained from $T$ by attaching a 2-handle $U$ along $\gamma_{0}$, and $\partial M$ is homeomorphic to the torus $S^{1} \times S^{1}$.

One can construct simple closed curves $\gamma_{1}$ and $\gamma_{2}$ in $\partial T-U$ that correspond respectively to the words $a^{p}$ and $a^{r} b^{s}$. Moreover this can be done so that $\partial M-\left(\gamma_{1} \cup \gamma_{2}\right)$ is connected, $\gamma_{1}$ and $\gamma_{2}$ intersecting in a single crossing point.

To see this, we use the techniques of [3]. Construct in $S^{2}$ a faithfully embedded $a$-syllable graph with three syllables whose exponents are $p, p$, and $r$, respectively, and a faithfully embedded $b$-syllable graph with two syllables whose exponents are $q$ and $s$. Note that this is possible since the gaps on 
the syllables $a^{p}$ and $b^{q}$ are $r$ and $s$ respectively. See Figure 1 for the construction. The ends of the $a$-syllables are indicated by 0,1 , and 2 , and those of the $b$-syllables by 0 and 2 . Connect the syllable ends with arcs as shown. In constructing $T$, we obtain simple closed curves $\gamma_{0}, \gamma_{1}$, and $\gamma_{2}$ in $\partial T$ corresponding, respectively, to the words $a^{p} b^{q}, a^{p}$, and $a^{r} b^{s}$. Moreover $\gamma_{1}$ and $\gamma_{2}$ intersect in the point $Q$, which is clearly a crossing point. Attaching the 2-handle $U$ to $T$ along $\gamma_{0}$ gives us the manifold $M$ with the curves $\gamma_{1}$ and $\gamma_{2}$ in $\partial M-U$ and intersecting at $Q$.

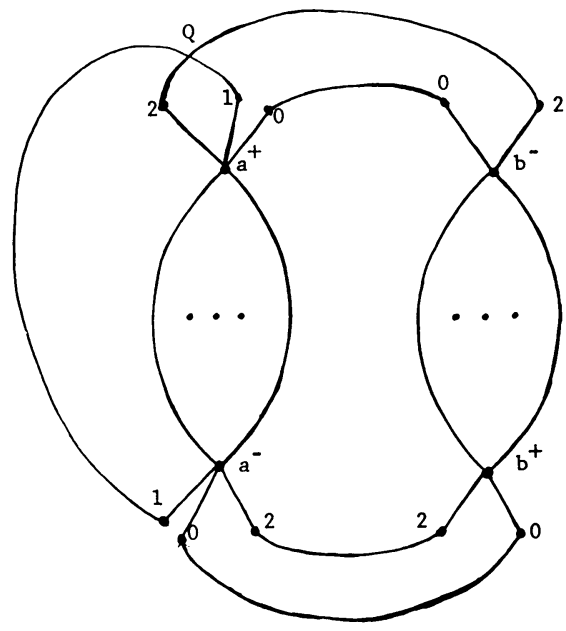

Figure 1

We show that $\gamma_{1} \cup \gamma_{2}$ does not separate $\partial M$. Let

$$
\psi_{1}=\left\langle a, b \mid a^{p} b^{q}, a^{p}\right\rangle \text { and } \psi_{2}=\left\langle a, b \mid a^{p} b^{q}, a^{r} b^{s}\right\rangle .
$$

Then $K_{\psi_{1}}$ and $K_{\psi_{2}}$ are spines of closed manifolds-the former of a connected sum of two lens spaces (by the multiplication theorem [4]) and the latter of a lens space (see [4]). This means that each $M_{i}$ has a 2-sphere boundary; hence, each curve $\gamma_{i}$ does not separate $\partial M(i=1,2)$. It follows that $\gamma_{1}$ $\cup \gamma_{2}$ does not separate $\partial M$.

Let $\gamma$ be any nonseparating simple closed curve in $\partial M$. Since $\pi_{1}(\partial M)$ is abelian and is generated by $a^{p}$ and $a^{r} b^{s}$, we observe that $\gamma$ corresponds to the word $W=\left(a^{p}\right)^{m}\left(a^{r} b^{s}\right)^{n}$ in $\pi_{1}(M)$ for an appropriate choice of $m$ and $n((m, n)=1)$. Let $\widetilde{M}$ be a closed manifold obtained from $M$ by attaching a 2-handle $U$ to $M$ along $\gamma$ and then attaching a 3-cell to the 2-sphere boundary of the resulting manifold. Then $\pi_{1}(\tilde{M})$ is presented by $\tilde{\psi}=\langle a, b|$ $\left.a^{p} b^{q}, W\right\rangle$.

We show that $\tilde{M}$ is a lens space if and only if $|n|=1$. If $n=0$, then $(m, n)=1$ forces $m= \pm 1$ and $\widetilde{M}$ is a connected sum of two nontrivial lens spaces. If $|n|>1$, then $\pi_{1}(\widetilde{M})$ has a homomorphism onto the group present- 
ed by $\left\langle a, b \mid a^{p}=b^{q}=\left(a^{r} b^{s}\right)^{n}=1\right\rangle$. This group can be shown not to be cyclic (see $[1$, p. 71]).

If $|n|=1$, we assume $n=1$ and obtain $\tilde{\psi}=\left\langle a, b \mid a^{p} b^{q}, a^{m p+r} b^{s}\right\rangle$ with $K_{\psi}$ a spine of $\tilde{M}$. Thus, $\tilde{M}$ is a lens space. Let $\lambda$ be the order of $\pi_{1}(\widetilde{M})$. Then $\lambda=|p s-q(m p+r)|$. Thus, assuming that $M_{p, r, q, s}$ and $M_{p, r^{\prime}, q, s^{\prime}}$ are homeomorphic, we conclude that

$$
p s-q r-m p q=\epsilon\left(p s^{\prime}-q r^{\prime}-m^{\prime} p q\right)
$$

for $\epsilon= \pm 1$ and appropriate choices of $m$ and $m^{\prime}$. Hence,

$$
p\left(s-\epsilon s^{\prime}\right)-q\left(r-\epsilon r^{\prime}\right)=p q\left(m-\epsilon m^{\prime}\right),
$$

and the Theorem follows.

Corollary. There exists a compact orientable irreducible 3-mainfold which is not embeddable in $S^{3}$ but which is of the same homotopy type as a torus knot space.

Proof. Suppose that $M=M_{p, r, q, s}$ is embeddable in $S^{3}$. Then $\partial M$ is a torus $S^{1} \times S^{1}$ in $S^{3}$. Since $M$ is not a solid torus, it follows that $\mathrm{cl}\left(S^{3}-M\right)$ is a solid torus. Hence $S^{3}$ is obtainable from $M$ by attaching a 2-handle along some nonseparating simple closed curve in $\partial M$ and then attaching a 3 -cell to the resulting 2 -sphere boundary. Thus $p s-q r-m p q= \pm 1$ for some $m$, a condition that is violated for $p=5, q=3, r=1$, and $s=1$.

The author wishes to thank Professor Herbert C. Lyon for helpful discussions。

\section{REFERENCES}

1. H. S. M. Coxeter and W. O. J. Moser, Generators and relations for discrete groups, Springer-Verlag, Berlin and New York, 1972.

2. W. Magnus, A. Karrass and D. Solitar, Combinatorial group theory: Presentations of groups in terms of generators and relations, Pure and Appl. Math., vol. 13, Interscience, New York, 1966. MR 34 \#7617.

3. R. P. Osborne and R. S. Stevens, Group presentations corresponding to spines of 3-manifolds. I, Amer. J. Math. 96 (1974), 454-471.

4. R. S. Stevens, Classifications of 3-manifolds with certain spines, Trans. Amer. Math. Soc. 205 (1975), 151-156.

5. F. Waldhausen, Gruppen mit Zentrum und 3-dimensionale Mannigfaltigkeiten, Topology 6 (1967) 505-517. MR 38 \#5223. 48503

DEPARTMENT OF MATHEMATICS, UNIVERSITY OF MICHIGAN, FLINT, MICHIGAN 